\title{
Dynamic MR imaging of the skeletal muscle in young and senior volunteers during minimal synchronized neuromuscular electrical stimulation.
}

Xeni Deligianni ${ }^{\mathrm{a}, \mathrm{b}}$, Christopher Klenk ${ }^{\mathrm{c}}$, Nicolas Place ${ }^{\mathrm{d}}$, Meritxell Garcia ${ }^{\mathrm{e}}$, Michele Pansini ${ }^{\mathrm{f}}$, Anna Hirschmann ${ }^{\mathrm{g}}$, Arno Schmidt-Trucksäss ${ }^{\mathrm{c}}$, Oliver Bieri ${ }^{\mathrm{a}, \mathrm{b}}$, Francesco Santini ${ }^{\mathrm{a}, \mathrm{b}}$

${ }^{a}$ Department of Radiology, Division of Radiological Physics, University Hospital Basel, Petersgraben 4, Basel, Switzerland

${ }^{b}$ Department of Biomedical Engineering, University of Basel, Gewerbestrasse 14, Allschwil, Switzerland

${ }^{c}$ Department of Sport, Exercise and Health, Division Sports and Exercise Medicine, University of Basel, Birsstrasse 320, Basel, Switzerland

${ }^{d}$ Institute of Sport Sciences, University of Lausanne, Bâtiment Synathlon, Quartier UNIL Centre, 1015, Lausanne, Switzerland

${ }^{e}$ TMC - European Telemedicine Clinic - a Unilabs company, Torre Mapfre, C/Marina 16 - 18, 08005 Barcelona, Spain

${ }^{f}$ Ricerche Diagnostiche Srl, Largo Ignazio Ciaia, 13, Bari, Italy

${ }^{g}$ Department of Radiology, University Hospital Basel, Petersgraben 4, Basel, Switzerland

Original Submission to Magnetic Resonance Materials in Physics, Biology and Medicine before Peer Review

\section{Corresponding author:}

Xeni Deligianni

Division of Radiological Physics, Department of Radiology, University Hospital Basel, Petersgraben 4, 4031, Basel, Switzerland

E-mail: xeni.deligianni@unibas.ch, Phone: +41-61-556-5728

\section{ACKNOWLEDGMENTS}

This is a pre-print of an article published in Magnetic Resonance Materials in Physics, Biology and Medicine. The final authenticated version is available online at: https://doi.org/10.1007/s10334-019-00787-7.

This work was supported by the Swiss Foundation for Research on Muscle Diseases (SSEM-FSRMM) and Swiss National Science Foundation (grant Nr. 172876). 


\section{ABSTRACT}

\section{Object}

Neuromuscular electrical stimulation (NMES)-induced isometric contraction is feasible during MRI and can be combined with acquisition of volumetric dynamic MR data, in a synchronous and controlled way. Since NMES is a potent resource for rehabilitation, MRI synchronized with NMES presents a valuable validation tool. Our aim was to show how minimal NMES-induced muscle contraction characterization, as evaluated through phase contrast MRI, differs between senior and young volunteers.

Materials and Methods

Simultaneous NMES of the quadriceps muscle and phase contrast imaging were applied at $3 \mathrm{~T}$ to 11 senior $(75 \pm 3$ years) and 6 young volunteers ( $29 \pm 7$ years). A current sufficient to induce muscle twitch without knee extension was applied to both groups.

Results

Strain vectors were extracted from the velocity fields and strain datasets were compared with non-parametric tests and descriptive statistics. Strain values were noticeably different between both groups at both current intensities and significant differences were observed in the regions of interest between the two electrodes.

\section{Discussion}

In conclusion, NMES-synchronized MRI could be successfully applied in senior volunteers with strain results clearly different from the younger volunteers. Also, differences within the senior group were detected both in the magnitude of strain and in the position of maximum strain pixels.

Keywords

phase contrast sequence; age; strain; quadriceps muscle; electrical muscle stimulation 


\section{INTRODUCTION}

The combination of MRI and neuromuscular electrical stimulation (NMES) not only gives information about the magnitude of the muscle response, but also localized feedback about how each part of the muscle reacts [1, 2]. NMES involves the application of a series of intermittent stimuli to superficial skeletal muscles to trigger visible muscle contractions due to the activation of the intramuscular nerve branches [3]. It can be controlled by adjusting the waveform, frequency and amplitude of stimulation [3] and induces a synchronous activation of the motor units. In combination with imaging, this enables the direct assessment of muscle kinematics through MRI, by synchronizing the MR-data acquisition with NMES as recently presented [4]. This method offers three-dimensional data and direct insights into the muscle contraction capabilities in a completely non-invasive way. It is a low-cost and easily-applicable solution and results significantly depend on the applied stimulation current [4]. Although NMES-responses have been investigated with MRI in the past $[1,2,5]$, an extensive evaluation of what to expect as a baseline in the MR-based parameters (e.g. normal velocity, displacement maps, strain, strain rate, etc.) and how these parameters can change because of physiological and pathological processes is still missing. Other existing approaches offer data acquisition before and after the scan [2] or a synchronization process that comes from the sequence and not the stimulator [6]. In addition, these methods only focus on $\mathrm{T}_{2}$ mapping $[2,5]$ or ${ }^{31} \mathrm{P}$ spectra acquisition [5, 6]. Yet, the contraction response of a muscle is important and complimentary to characterize its mechanical/elastic capacities.

Comparably to existing approaches for voluntary exercise protocols $[7,8]$, the suggested method uses velocity information acquired with phase contrast MRI (PC MRI) and provides dynamic muscle images in a similar way to cardiac imaging [9]. Further quantitative evaluation of PC images yields strain maps [7, 10, 11]. While voluntary contraction follows the Henneman size principle (i.e., small motor units are recruited at lower force levels as compared to larger motor units)[12, 13], standard NMES induces a non-selective and mostly superficial random motor unit recruitment, allowing type II muscle fiber recruitment even at low force levels [3, 12, 14-16].

Age-related changes of the skeletal muscle tissue are associated with a reduction of muscle mass (sarcopenia), which is closely associated with a reduced number of motor units [17-19]. It is well accepted that type II (fast-twitching) fibers are the most affected ones [18, 20, 21], which results into muscle fiber grouping, i.e. the reorganization of the remaining fibers in larger motor units $[17,21]$. For these reasons, employing NMES as a tool to study muscle fiber alterations in aged muscle can be particularly interesting. 
The aim of this study was to investigate velocity imaging in the quadriceps muscle through NMES-synchronized MRI and evaluate the potential differences between senior and young volunteers. While physiological differences are expected between these two population groups [7], the suitability of the proposed stimulation and imaging protocols to highlight such differences cannot be presumed and is thus the primary endpoint of this study.

\section{MATERIAL AND METHODS}

The study was approved by the local ethics committee and written informed consent was obtained from all individual participants included in the study. Volunteers with a history of heart or kidney disease, cancer and muscle pathology or any operation on the examined lower extremity within the past five years were excluded. A total of 11 healthy senior (mean age: $74.9 \pm 3.4$ years, range 70-82; mean height: $170 \pm 8 \mathrm{~cm}, 6$ male, 5 female) and 6 healthy young volunteers (age: $29.0 \pm 6.5$ years, (21-35), height: $179 \pm 8 \mathrm{~cm}, 6$ male) were included.

\section{Experimental setting}

An InTENSity Twin Stim III TENS and NMES Combo (Current Solutions LLC, Austin, TX) was used for the stimulation of the quadriceps muscle and $5.1 \times 8.9 \mathrm{~cm}^{2}$ rectangular self-adhesive gel-based NMES electrodes (TENSUnits) were attached to the muscle belly as described in [4]. The electrodes were placed at $15 \mathrm{~cm}$ distance from each other and $12 \mathrm{~cm}$ from the center of the knee joint on the vastus lateralis (VL). The young volunteers were scanned once with the stimulation level set to $18 \mathrm{~mA}$, which was sufficient to achieve muscle twitching without knee extension [4]. For the senior volunteers, the stimulation was applied first at a minimum level to induce a visible contraction within their comfort levels and with the maximum limit set to $22 \mathrm{~mA}$. Five minutes after the maximum applied level, an additional acquisition was obtained at $18 \mathrm{~mA}$ for comparison.

A monopolar square wave with frequency set to 150 pulses/s and pulse duration set to $0.3 \mathrm{~ms}$ was used for stimulation. The plateau of each contraction lasted $1 \mathrm{~s}$ (i.e., $1 \mathrm{~s}$ ramp time, $1 \mathrm{~s}$ plateau, $1 \mathrm{~s}$ ramp down, $2 \mathrm{~s}$ relaxation). A second waveform, generated at the beginning of every stimulation cycle, was used for triggering of the MRI acquisition.

MR acquisition 
The acquisitions were performed on a 3T clinical MRI scanner (MAGNETOM Prisma, Siemens Healthcare, Erlangen, Germany) in the same way and with the same hardware setup as previously described [4]. For the experimental setup, the NMES device was used to periodically stimulate the quadriceps muscle and was synchronized with a single-slice three-directional gradient echo phase contrast (PC) MRI acquisition [4]. A threedirectional gradient echo PC velocity encoding sequence was applied. MR acquisitions were performed on a parasagittal slice (through VL and vastus intermedius (VI) muscles) with a spatial resolution of $2.3 \times 2.3 \times 5$ mm ${ }^{3}$ and a temporal resolution of $42 \mathrm{~ms}$. The velocity encoding was $25 \mathrm{~cm} / \mathrm{s}$ (repetition time (TR)/echo time (TE) = 10.6/7.21 ms, bandwidth/pixel $=400 \mathrm{~Hz} / \mathrm{Px}$, flip angle $=10^{\circ}$, field-of-view $=225 \mathrm{x} 300 \mathrm{~mm}^{2}, 1 \mathrm{k}$-space line per segment, acquisition time $5 \mathrm{~min}$ ) and 94 temporal phases were acquired. In total, during the whole image acquisition time approximately 60 contractions were induced.

\section{Data processing}

The velocity images were elaborated offline with Matlab (The Mathworks, Inc., Natick, MA, USA). Strain tensors were extracted from the velocity fields as described in [7, 10, 22] and subsequently diagonalized to obtain the strain

eigenvalues ${ }^{e_{1}}$. As the acquisition was limited to a single slice, only the in-plane strain tensors could be extracted from the velocity field.

The post-processing analysis was performed initially for the VL and VI and then four different regions-of-interest (ROI) were selected equidistantly covering both VL and VI muscles proximally to distally in respect to the knee. ROIs 2 \& 3 were located approximately in between the two stimulation electrodes (see Figure 1). For every time frame, the spatial median values were calculated (to account for the skewness of the statistical distribution of the values inside the ROIs). Temporal local maximum values were calculated for the strain over each ROI [4].

In addition to the magnitude of the deformation as described by the strain values, temporal information (i.e., the rate of reaching the maximum response) was also extracted from the datasets. This information was obtained in terms of "increase rate" of the strain following the stimulus and was calculated as the slope of the line connecting the beginning of the contraction (defined as the point of maximum curvature of the strain curve) and the maximum point of the same curve. This parameter was defined as strain increase rate and it was descriptively evaluated through maps. 
Statistical analysis

Comparison with a significance level of 0.05 was performed between the independent groups of the results from the senior volunteers (SV) for $18 \mathrm{~mA}$ and $22 \mathrm{~mA}$ (SV18 and SV22) versus the results of the young volunteers (YV) at $18 \mathrm{~mA}$ (YV18). The comparison was performed for all four ROIs. The internal control for distribution normality was performed with qualitative histogram visualization. Given the low number of participants non-parametric statistics were applied; since the distributions were not all normal and the number of samples was small, two-sided Wilcoxon rank sum test was used. Statistical analysis was performed with Matlab (ranksum function). Due to the small number of volunteers, no statistical analysis between genders was performed, but the results of the male volunteers were analyzed separately to ensure there was no considerable bias (6 young versus 6 senior participants).

\section{RESULTS}

All six YV were scanned successfully with the stimulation current set at $18 \mathrm{~mA}$. Dynamic PC images were successfully acquired from SV at $18-22 \mathrm{~mA}$. For the majority of SV (9 out of 11), a current amplitude of $22 \mathrm{~mA}$ had to be applied to achieve a similar muscle twitch as compared to $18 \mathrm{~mA}$ in the YV. Two SV were scanned at 20 $\mathrm{mA}$ instead of $22 \mathrm{~mA}$, because the lower current already achieved sufficient muscle twitch. Since these were only two cases, the results from the scan at $20 \mathrm{~mA}$ of these two SV were not analyzed, but only the ones at $18 \mathrm{~mA}$.

In general, the velocity averaged over e.g. the VL as a function of time presents two pronounced peaks one at the beginning of the contraction and one at the moment of the release of the muscle [4]. In Figure 2, some exemplary velocity vector maps from the beginning of the contraction are presented. The three-dimensional colored velocity vectors from the VL were overlayed on an anatomical image of the thigh. In the senior volunteers, the contraction peak occasionally appeared at a later time frame than in the younger volunteers (i.e. around the $40^{\text {th }}$ frame instead of the $30^{\text {th }}$ frame).

The principal strain maps were calculated, and the temporal evolution of strain was analyzed for the VL and VI (see Figure 3). As expected, we observed a response to the stimulation in both muscles, the VL and the VI, yet the response in the VL was stronger. When applying a lower current (i.e., $18 \mathrm{~mA}$ ) to the senior volunteers, there was no discernible response in the VI. Moreover, for the younger volunteers, the strain reaches a maximum value faster than for senior volunteers (see Figure 3). 
The strain values in both VL and VI were summarized for the four different ROIs (ROI1-4: proximal to distal). As expected, for the central ROIs $2 \& 3$, that are located approximately between the electrodes, the strain values had significantly lower values for the senior in comparison to the younger volunteers (Figure 4).

Finally, the analysis of the spatial distribution of the strain increase rate showed various different patterns. Figure 5 shows three out of five cases of senior volunteers with no distinct regions characterized by higher values (Figure 5d, 5f, 5h) and three out of four cases, in which those regions were very small (Figure 5g, 5i, 5j). On the contrary, some distinct connected regions with higher rates were found for all young volunteers and four of the senior volunteers (Figure 5a, 5b, 5c,5e).

\section{Statistical analysis}

The results of the two-sided Wilcoxon rank sum test are presented in Table 1. The comparison was performed for the four ROIs of both VL and VI. The differences between young and senior volunteers for contractions at $18 \mathrm{~mA}$ were significant for the 2 central ROIs $(p=0.002)$. Significant differences of strain values from young volunteers and senior volunteers at $22 \mathrm{~mA}$ were found for ROI 3 ( $p=0.012)$, which is in agreement with the boxplot visualization (see Figure 4).

Comparison of male volunteers (6 young and 6 senior) revealed different range and $p$-values (see Table 1), however, the result of the test remained the same.

\section{DISCUSSION}

The aim of this study was to investigate velocity imaging in the quadriceps muscle through NMES-synchronized MRI and evaluate the potential differences between senior and young volunteers. Significant differences in skeletal muscle contraction parameters (i.e., principal strain) were assessed with PC MRI between healthy young ( $<35$ years old) and senior ( $>70$ years old) volunteers. It was also shown that the difference of strain values between the young and senior volunteers, at the same stimulation current, was larger at the ROIs between the two NMES electrodes.

The significant difference in strain values between the two age groups is in agreement with Sinha et al. who showed differences not in strain but in strain rate maps calculated from PC images in senior and younger volunteers (78 years vs 28 years) during voluntary contractions [7]. While this agreement seems straightforward, the results 
presented in this work could not be simply deduced from similar data acquired during voluntary contraction, since the two types of exercise are fundamentally different. In our case, the difference between the two populations could be attributed to stiffer muscle elasticity with increasing age [17, 18, 23]. A reduced number and size of mainly type II muscle fibers in the seniors might contribute to a difference in strain values between young and senior individuals as well, since NMES allows type II muscle fiber recruitment even at low force levels [17, 18, 20].

In addition, the spatial distribution of the parameter of the strain curve, defined here as "strain increase rate", was examined. This parameter intimately relates to the strain rate, determined as the temporal derivative of strain, but it still depends on the reference state. For linearly increasing strain curves, the two parameters should be approximately alike. However, the muscle response to electrical stimulation is not linear (i.e., the force, the magnitude of stretching, etc) and often not monotonic and thus this assumption is typically not valid. This was the case especially in the senior volunteers, who overall proved to be less responsive to the same stimulation current.

In the present study, we observed a faster response of higher amplitude, that "activated" a larger area of the most superficial muscles in younger compared to the senior volunteers. This observation can be used as a potential marker to show efficacy and improvement of NMES training protocols in the aged muscle. Furthermore, within the SV group some responses were similar to the ones of the YV group. A next step would be to investigate whether this fact depends on special characteristics of one's physical status, which has to be characterized with other parameters such as external force measurements.

Clinical applications of the presented method include a variety of pathological muscle conditions sensitive to fiber type II atrophy such as chronic obstructive pulmonary disease [24], or chronic steroid myopathy [3, 25], and agerelated diseases such as sarcopenia [26]. It can also be applied to competitive or elderly people who need to train fast fibers with low effort $[15,16]$.

Due to the limited number of volunteers in this study, the group of senior volunteers was considered as one single group. However, there was a variability in the physical condition of the subjects, since some of the senior volunteers performed vigorous training on a regular basis. For better differentiation, the volunteers would have to be grouped more strictly according to their physical condition and training habits, which can be the subject of future investigation. 
Finally, one technical restriction of the current study is that there was no comparison of the calculated strain with the force output. In part, this was due to the lack of a suitable measurement equipment at our institution. Yet the choice of using a stimulation current that only generates a visible twitch of the muscle without noticeable knee extension was dictated by the strong discomfort associated with NMES at higher force outputs [3], which makes the detectability of physiological differences at minimal stimulation intensity very relevant for the compliance and comfort of a potential patient. Nevertheless, it would be interesting to compare strain for the same force output in future investigations.

In conclusion, strain measurements with MRI of NMES-induced muscle contraction show age-related differences between healthy volunteers above 70 and below 35 years old. The differences were more significant when the same stimulation current was used for young and senior subjects. Moreover, there were prominent differences not only in the strain magnitude, but also in the temporal rate of strain and variable for different muscle regions. Despite these physiological inter-individual differences, the data shown here may be used as a preliminary data baseline for a more accurate and detailed assessment of muscle function disturbances.

\section{ACKNOWLEDGMENTS}

This work was supported by the Swiss Foundation for Research on Muscle Diseases (SSEM-FSRMM) and Swiss National Science Foundation (grant Nr. 172876).

\section{AUTHORS'CONTRIBUTION}

XD: Study conception and design, Acquisition of data, Analysis and interpretation of data, Drafting of manuscript, Critical revision

CK: Study conception and design, Drafting of manuscript

NP: Analysis and interpretation of data, Drafting of manuscript, Critical revision

MG: Study conception and design, Acquisition of data, Drafting of manuscript

MP: Study conception and design, Drafting of manuscript

AH: Study conception and design, Drafting of manuscript, Acquisition of data

AS-T: Study conception and design, Drafting of manuscript

OB: Study conception and design, Drafting of manuscript 
FS: Study conception and design, Acquisition of data, Analysis and interpretation of data, Drafting of manuscript, Critical revision

Conflict of Interest:

The authors declare that they have no conflict of interest.

Ethics:

The study was approved by the local ethics committee and written informed consent was obtained from all individual participants included in the study

\section{REFERENCES}

1. Adams GR, Harris RT, Woodard D, Dudley GA (1993) Mapping of electrical muscle stimulation using MRI. J Appl Physiol 74:532-537.

2. Meyerspeer M, Mandl T, Reichel M, Mayr W, Hofer C, Kern H, Moser E (2008) Effects of functional electrical stimulation in denervated thigh muscles of paraplegic patients mapped with T2 imaging. Magma N Y N 21:219-226.

3. Nicola A. Maffiuletti (2010) Physiological and methodological considerations for the use of neuromuscular electrical stimulation. Eur J Appl Physiol 110:223-234.

4. Deligianni X, Pansini M, Garcia M, Hirschmann A, Schmidt-Trucksäss A, Bieri O, Santini F (2017) Synchronous MRI of muscle motion induced by electrical stimulation. Magn Reson Med 77:664-672.

5. Jubeau M, LE Fur Y, Duhamel G, Wegrzyk J, Confort-Gouny S, Vilmen C, Cozzone PJ, Mattei JP, Bendahan D, Gondin J (2015) Localized metabolic and t2 changes induced by voluntary and evoked contractions. Med Sci Sports Exerc 47:921-930.

6. Vanderthommen M, Duteil S, Wary C, Raynaud JS, Leroy-Willig A, Crielaard JM, Carlier PG (2003) A comparison of voluntary and electrically induced contractions by interleaved 1H- and 31P-NMRS in humans. J Appl Physiol 94:1012-1024.

7. Sinha U, Malis V, Csapo R, Moghadasi A, Kinugasa R, Sinha S (2015) Age-related differences in strain rate tensor of the medial gastrocnemius muscle during passive plantarflexion and active isometric contraction using velocity encoded MR imaging: Potential index of lateral force transmission. Magn Reson Med 73:1852-1863.

8. Sinha S, Shin DD, Hodgson JA, Kinugasa R, Edgerton VR (2012) Computer-controlled, MR-compatible footpedal device to study dynamics of the muscle tendon complex under isometric, concentric, and eccentric contractions. J Magn Reson Imaging 36:498-504.

9. Drace JE, Pelc NJ (1994) Measurement of skeletal muscle motion in vivo with phase-contrast MR imaging. J Magn Reson Imaging 4:157-163.

10. Wedeen VJ (1992) Magnetic resonance imaging of myocardial kinematics. Technique to detect, localize, and quantify the strain rates of the active human myocardium. Magn Reson Med 27:52-67. 
11. Lee H-D, Finni T, Hodgson JA, Lai AM, Edgerton VR, Sinha S (2006) Soleus aponeurosis strain distribution following chronic unloading in humans: an in vivo MR phase-contrast study. J Appl Physiol 100:20042011.

12. Gregory CM, Bickel CS (2005) Recruitment Patterns in Human Skeletal Muscle During Electrical Stimulation. Phys Ther 85:358-364.

13. Henneman E, Somjen G, Carpenter DO (1965) Functional significance of cell size in spinal motoneurons. J Neurophysiol 28:560-580.

14. Bickel CS, Gregory CM, Dean JC (2011) Motor unit recruitment during neuromuscular electrical stimulation: a critical appraisal. Eur J Appl Physiol 111:2399.

15. Babault N, Cometti G, Bernardin M, Pousson M, Chatard J-C (2007) Effects of electromyostimulation training on muscle strength and power of elite rugby players. J Strength Cond Res 21:431-437.

16. Kamen G, Knight CA (2004) Training-related adaptations in motor unit discharge rate in young and older adults. J Gerontol Biol Sci Med Sci 59:1334-1338.

17. Porter MM, Vandervoort AA, Lexell J (1995) Aging of human muscle: structure, function and adaptability. Scand J Med Sci Sports 5:129-142.

18. Lexell J (1995) Human aging, muscle mass, and fiber type composition. J Gerontol A Biol Sci Med Sci 50 Spec No:11-16.

19. Lexell J, Taylor CC, Sjöström M (1988) What is the cause of the ageing atrophy? Total number, size and proportion of different fiber types studied in whole vastus lateralis muscle from 15- to 83-year-old men. J Neurol Sci 84:275-294.

20. Nilwik R, Snijders T, Leenders M, Groen BBL, van Kranenburg J, Verdijk LB, van Loon LJC (2013) The decline in skeletal muscle mass with aging is mainly attributed to a reduction in type II muscle fiber size. Exp Gerontol 48:492-498.

21. Andersen JL (2003) Muscle fibre type adaptation in the elderly human muscle. Scand J Med Sci Sports 13:4047.

22. Finni T, Hodgson JA, Lai AM, Edgerton VR, Sinha S (2006) Muscle synergism during isometric plantarflexion in achilles tendon rupture patients and in normal subjects revealed by velocity-encoded cine phase-contrast MRI. Clin Biomech 21:67-74.

23. Ochala J, Frontera WR, Dorer DJ, Van Hoecke J, Krivickas LS (2007) Single skeletal muscle fiber elastic and contractile characteristics in young and older men. J Gerontol Biol Sci Med Sci 62:375-381.

24. Gosker HR, Engelen MPKJ, van Mameren H, van Dijk PJ, van der Vusse GJ, Wouters EFM, Schols AMWJ (2002) Muscle fiber type IIX atrophy is involved in the loss of fat-free mass in chronic obstructive pulmonary disease. Am J Clin Nutr 76:113-119.

25. Kanda F, Okuda S, Matsushita T, Takatani K, Kimura KI, Chihara K (2001) Steroid myopathy: pathogenesis and effects of growth hormone and insulin-like growth factor-I administration. Horm Res 56 Suppl 1:24-28.

26. Sanabria SJ, Martini K, Freystätter G, Ruby L, Goksel O, Frauenfelder T, Rominger MB (2019) Speed of sound ultrasound: a pilot study on a novel technique to identify sarcopenia in seniors. Eur Radiol 29:3-12.

TABLES 


\begin{tabular}{|c|c|c|c|c|c|c|c|}
\hline & \multicolumn{3}{|c|}{ YV18-SV18 } & \multicolumn{3}{|c|}{ YV18-SV22 } & \multirow[b]{2}{*}{$\mathrm{H}$} \\
\hline & Gender & $R$ & p-value (CI95\%) & $\mathrm{H}$ & $R$ & p-value (CI95\%) & \\
\hline ROI-1 & $\mathrm{m} / \mathrm{f}$ & 71 & 0.089 & 0 & 52.5 & 0.634 & 0 \\
\hline ROI-2 & $\mathrm{m} / \mathrm{f}$ & 83. & 0.002 & 1 & 59 & 0.212 & 0 \\
\hline ROI-3 & $\mathrm{m} / \mathrm{f}$ & 5 & 0.002 & 1 & 68.5 & 0.012 & 1 \\
\hline ROI-4 & $\mathrm{m} / \mathrm{f}$ & $\begin{array}{l}83 . \\
5\end{array}$ & 0.142 & 0 & 56 & 0.372 & 0 \\
\hline & & 69 & & & & & \\
\hline ROI-1 & $\mathrm{m}$ & 51 & 0.065 & 0 & 38.5 & 0.701 & 0 \\
\hline ROI-2 & $\mathrm{m}$ & 55 & 0.011 & 1 & 35 & 0.892 & 0 \\
\hline ROI-3 & $\mathrm{m}$ & 57 & 0.002 & 1 & 47.5 & 0.048 & 1 \\
\hline ROI-4 & $\mathrm{m}$ & 49 & 0.128 & 0 & 40.5 & 0.455 & 0 \\
\hline
\end{tabular}

Table 1. Results of Wilcoxon rank sum test for strain between young volunteers' values at $18 \mathrm{~mA}$ (YV18) and senior volunteers at 18 and $22 \mathrm{~mA}$ (SV18 and SV22) ( $R$ : order of significance, $p$ : $p$-value of the test, $\mathrm{H}=0$ : the null hypothesis cannot be rejected at the $5 \%, \mathrm{H}=1$ indicates that the null hypothesis can be rejected at the $5 \%$ level). 


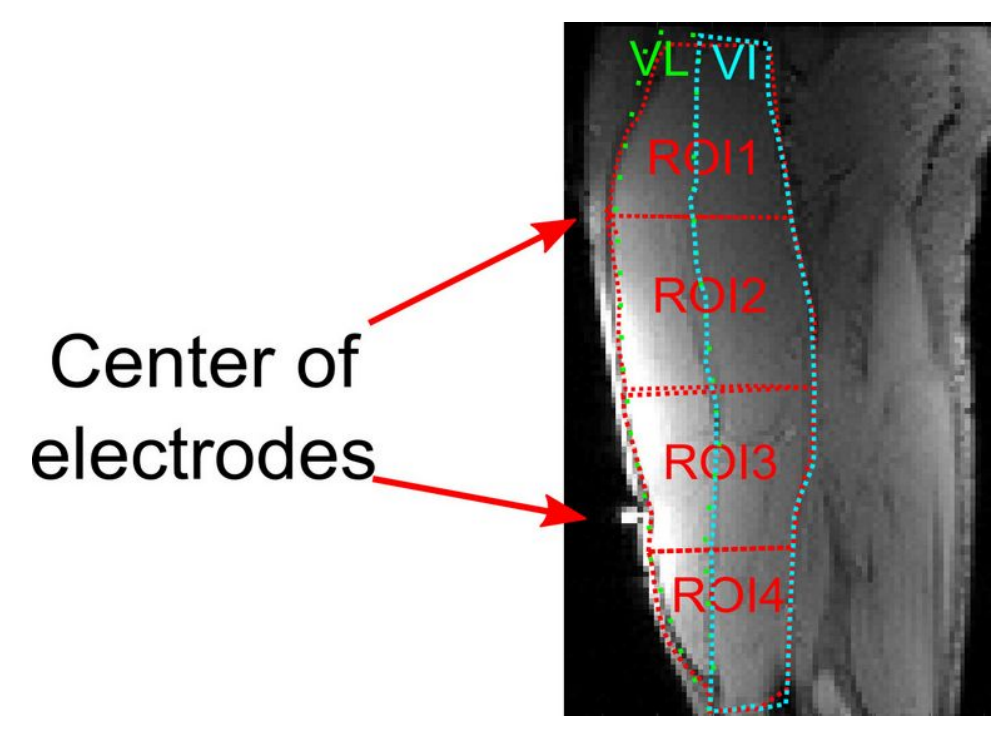

Fig. 1 Description of the placement of the regions of interest in respect to the muscles and to the electrodes position 


\section{YOUNG VOLUNTEERS}

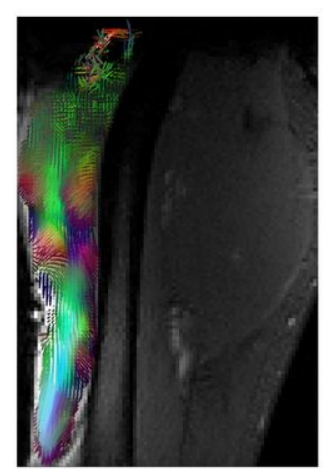

YV, \#35

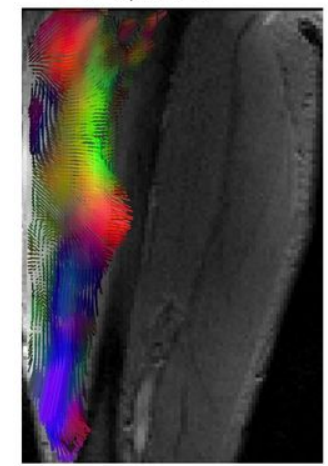

YV, \#32
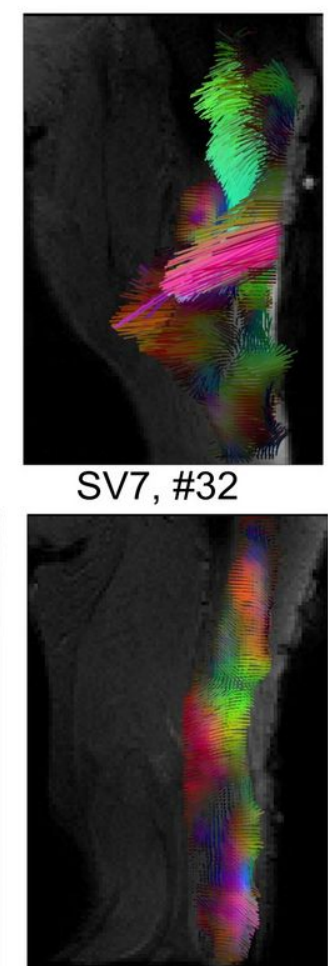

SV2, \#36

\section{SENIOR \\ VOLUNTEERS}

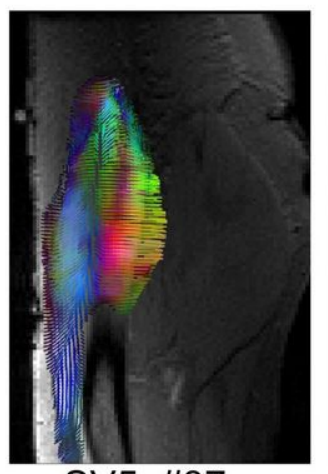

SV5, \#37

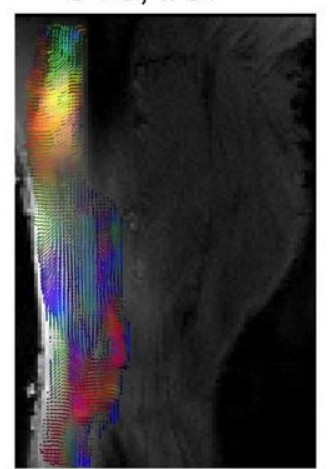

SV6, \#40

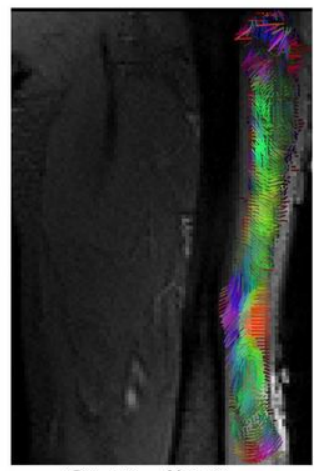

SV8, \#42

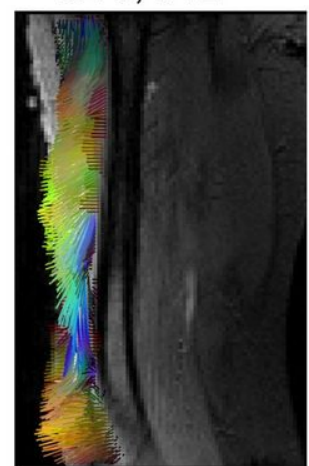

SV11, \#35

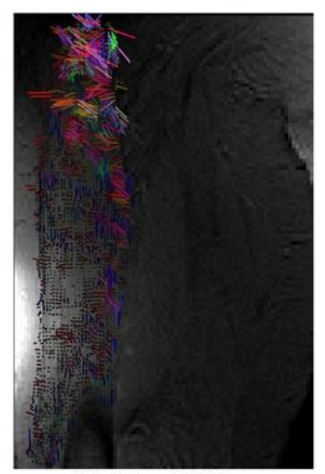

SV4, \#31

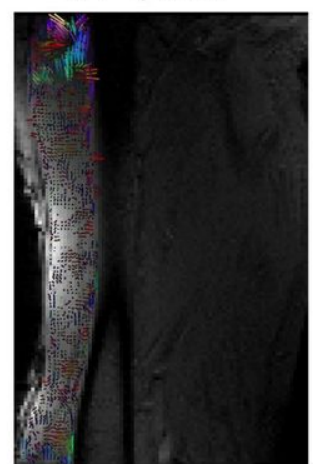

SV9, \#42

Fig. 2 Three-dimensional velocity maps at the beginning of the contraction plateau for ten exemplary cases of young and senior volunteers. The colored vectors are normalized according to the maximum. Velocity vectors inside the quadriceps are represented as color-coded lines (i.e., as in the cube) according to the direction (red: phase encoding direction, blue: readout direction, green: slice direction). The difference in homogeneity of the contraction can be seen in the color-coding 

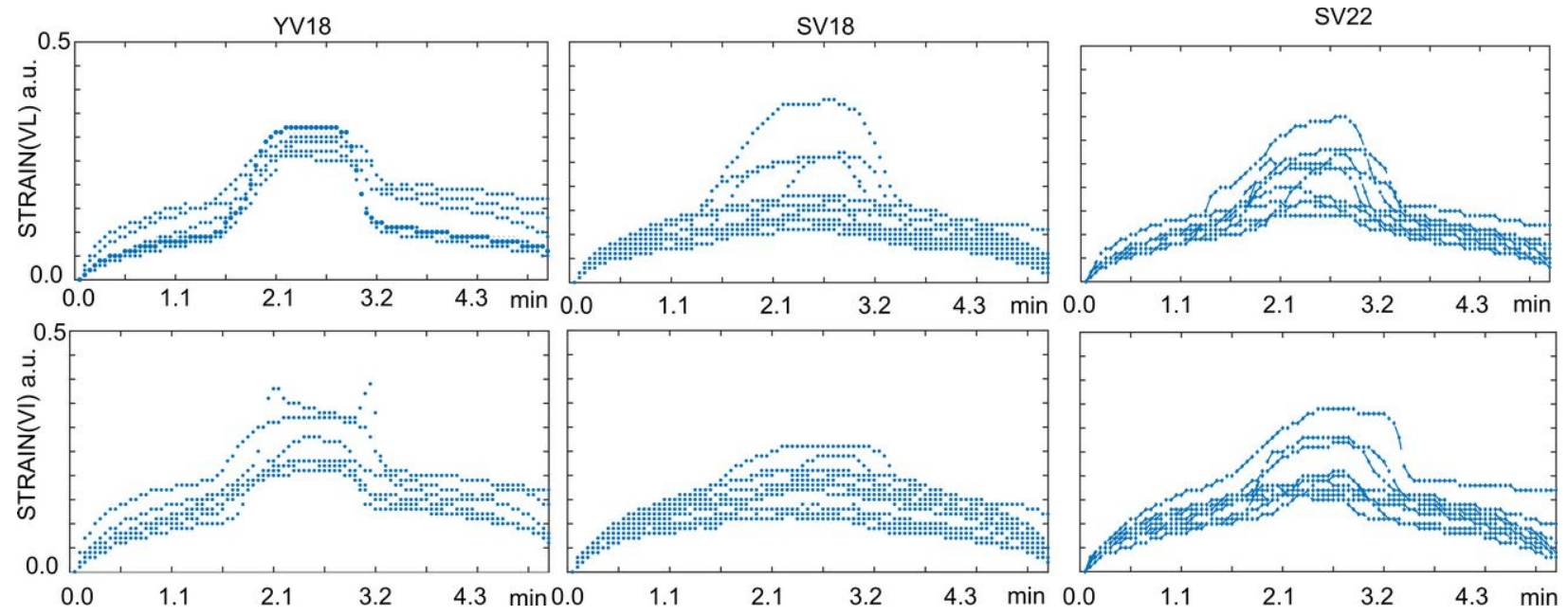

Fig. 3 Temporal evolution of strain calculated for the vastus lateralis (VL, upper row) and vastus intermedius (VI, lower row) at every time frame given in arbitrary units (a.u.). Results are given for both young (YV18: young volunteers at $18 \mathrm{~mA}$ ) and senior volunteers (SV18: senior volunteers at $18 \mathrm{~mA}$, SV22: senior volunteers at $22 \mathrm{~mA}$ ). Large variability both in maximum values and breadth of the curve can be observed 

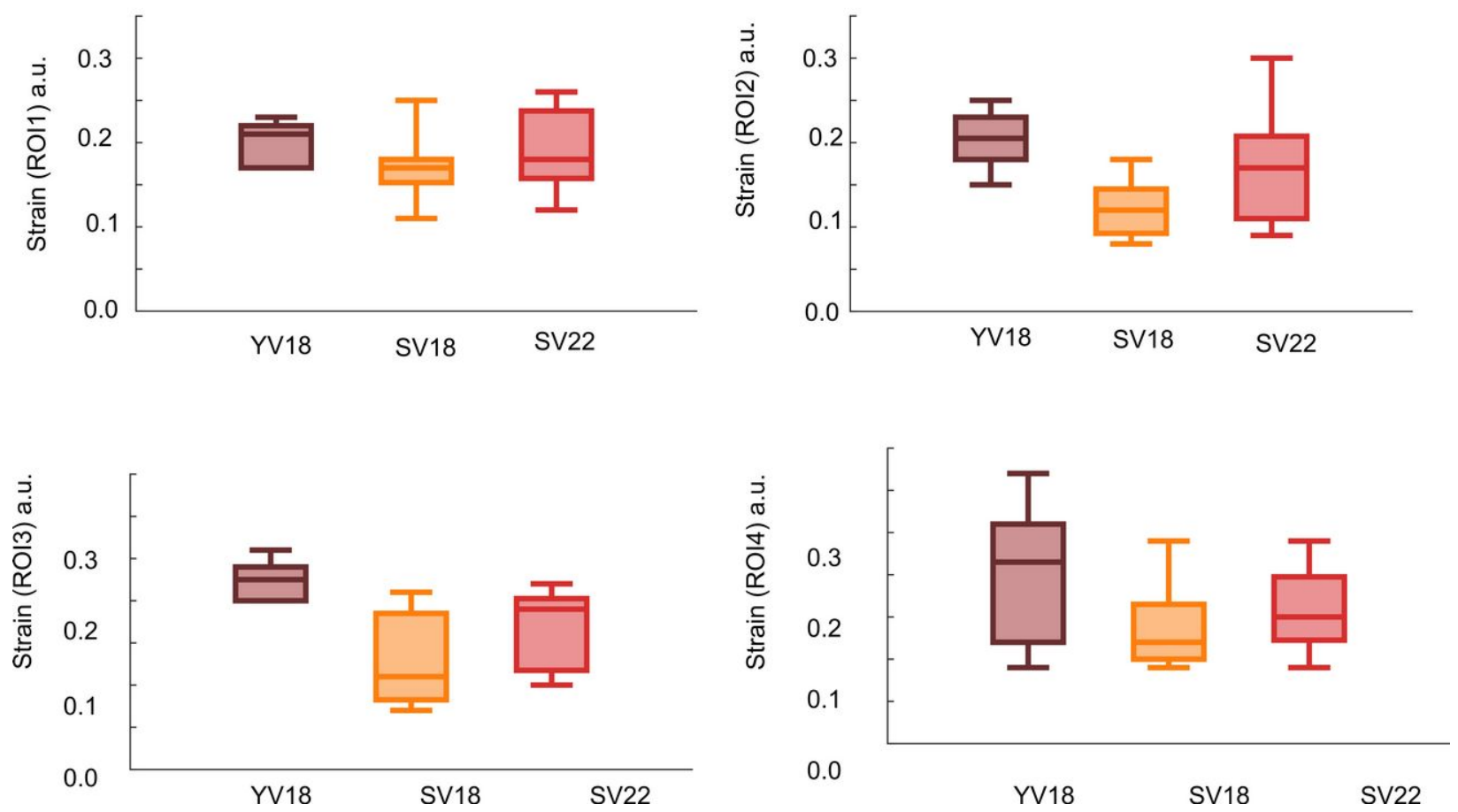

Fig. 4 Boxplots of strain values of the young volunteers (YV) at $18 \mathrm{~mA}$ (YV18, left boxplot), and of the senior volunteers (SV) at $18 \mathrm{~mA}$ and $22 \mathrm{~mA}$ (SV18, central boxplot and SV22, right boxplot) averaged over four different ROIs of the vastus lateralis from proximal to distal $\left(\mathrm{ROI}_{1}\right.$ to $\mathrm{ROI}_{4}$ see Fig. 1) 


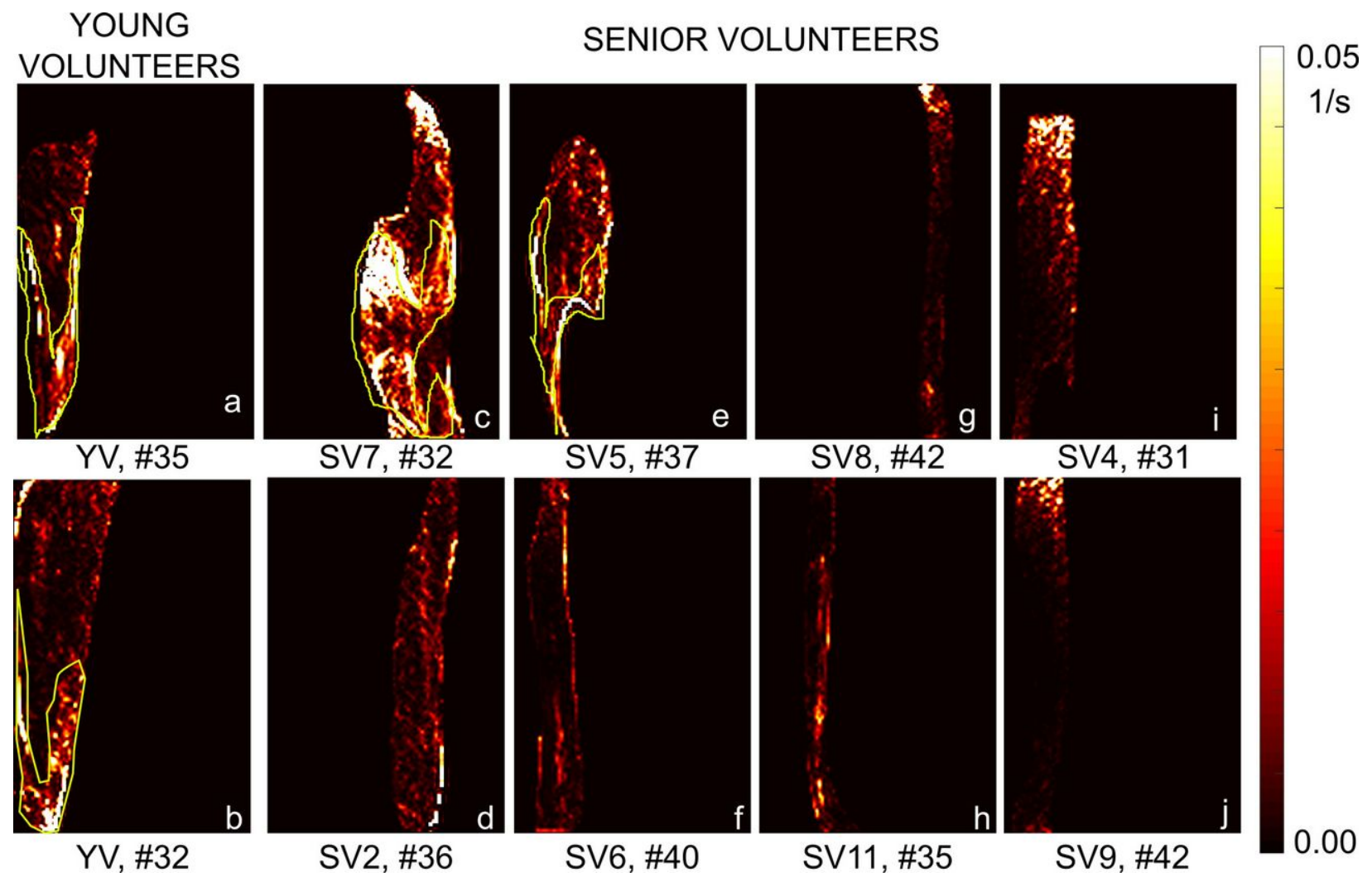

Fig. 5. Examples of strain increase rate maps of young volunteers (YV-left) and senior volunteers (SV-right). The respective number of the time frame is given on every image (YV/SV number, \# number of frame). A clear region with hyperintense values at the borders of the muscle (see delineation in green) can be identified in both young volunteers, but only in a limited number of two senior subjects 